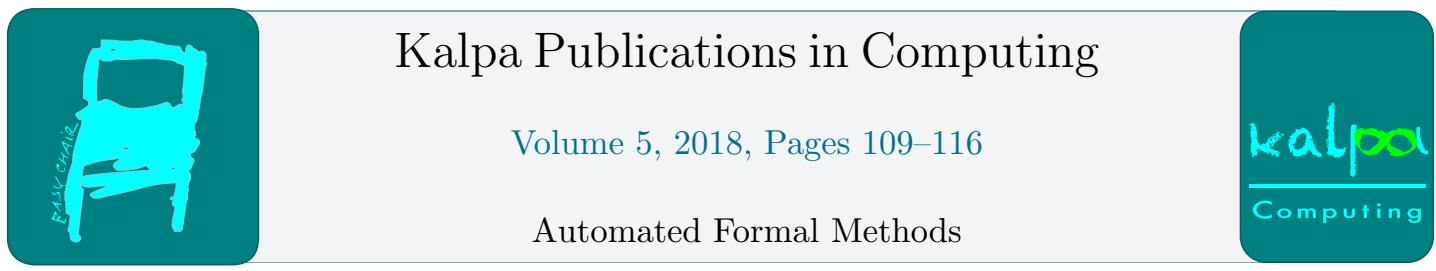

\title{
A Brief Introduction to the PVS2C Code Generator *
}

\author{
Natarajan Shankar \\ SRI International Computer Science Laboratory \\ 333 Ravenswood Avenue \\ Menlo Park, CA 94025 USA \\ shankar@csl.sri.com
}

\begin{abstract}
We present a brief tutorial on the PVS2C code generator for producing $\mathrm{C}$ code from an applicative fragment of the PVS specification language. This fragment roughly corresponds to a self-contained functional language. The tutorial covers the generation of $\mathrm{C}$ code for numeric data types and associated operations, arrays, recursive data types, and higherorder operations.
\end{abstract}

\section{Introduction}

Specification languages are meant to capture the "what" of computation while programming language express the "how". For this reason, a specification language need not be executable. However, many specification languages do contain executable sublanguages. Execution is useful for validating specifications, generating verified software and systems, and for performing large calculations within proofs. Code generation makes it possible to construct executable systems without having to formalize programming notations and their semantics within the specification language, or building special-purpose verification tools that target these programming languages.

The Prototype Verification System (PVS) is an interactive proof assistant with an expressive specification language based on higher-order logic. The type system admits predicate subtypes, dependent tuple, record, and function types, and recursive datatypes. The language also supports parametric theories. The expression language includes function application, lambda abstraction, quantification, conditional expressions, LET-binding, and record/tuple/function updates. The quantifier-free fragment of the language can be viewed as an applicative language.

There are two basic problems in mapping an applicative language to an imperative one. The first is that applicative semantics require copying on updates. This kind of copying can be

${ }^{*}$ This work was supported by NSF Grant CSR-EHCS(CPS)-0834810, NASA Cooperative Agreement NNA10DE73C, and by DARPA under agreement number FA8750-12-C-0284 and FA8750-16-C-0043. The views and conclusions contained herein are those of the authors and should not be interpreted as necessarily representing the official policies or endorsements, either expressed or implied, of NSF, NASA, DARPA or the U.S. Government.

N. Shankar and B. Dutertre (eds.), AFM17 (Kalpa Publications in Computing, vol. 5), pp. 109-116 
very expensive: sorting a 1000-element array can involve thousands of copies. It is therefore important to identify and exploit opportunities for in-place updates. The second problem is of course the execution of an applicative program can generate memory that is no longer referenced and needs to be garbage-collected. In PVS2C [1], reference counting is used to address both issues. In simple terms, an array can be updated in place when its reference count is one, and it can be garbage collected when its reference count drops to zero. Since reference cycles cannot be created when executing PVS, reference counting does ensure that no live references are collected and all dead references are garbage-collected. More strongly, references are released as soon as possible so as to maximize the opportunities for in-place updates. The execution of well-typed PVS expressions is safe: the only possible runtime errors are when the execution exhausts heap or stack space. Typechecking, particularly through the discharging of type correctness condition (TCC) proof obligations, ensures that there can be no buffer overflows, null dereferences, uncaught exceptions, division by zero, etc.

Though PVS2C targets the $\mathrm{C}$ programming language, the translation from PVS to $\mathrm{C}$ is factored through an intermediate representation (IR) that can be used to target other programming languages. The IR is based on A-normal form [2]. The pvs2ir operation translates PVS expressions into the IR. This translation basically involves flattening expressions to create variable bindings for subexpressions. The IR includes some type information to help track array sizes. The ir2c operation maps IR expressions into $\mathrm{C}$ by essentially converting the LETbindings into assignments. The PVS2C generator can be invoked as $\mathrm{M}-\mathrm{x}$ pvs-c-theory with the cursor on a theory in a .pvs file. The code generator generates a header and code file for the given theory as well as for any theories that are in the import chain. The code generator currently handles Boolean, numeric, record, tuple, recursive datatypes, and function types. Fixed width, uni-dimensional arrays are handled using $\mathrm{C}$ arrays, and the others are treated as function types. We are working on extending the translation to dependently sized array types and polymorphic types. We present a short tutorial on the use of the prototype implementation of PVS2C.

\section{A Small Example}

We first present a small example to illustrate the flow with the theory smallswap shown below. The type nat32 is a subtype that captures the $\mathrm{C}$ type uint_32. The pvs2ir translator uses the Common Lisp ground evaluator [3] to evaluate such expressions. This also holds for the numrows parameter. The type smallarray represents an array of size 5 . The swap operation is defined to exchange $A(i)$ with $A(j)$. 


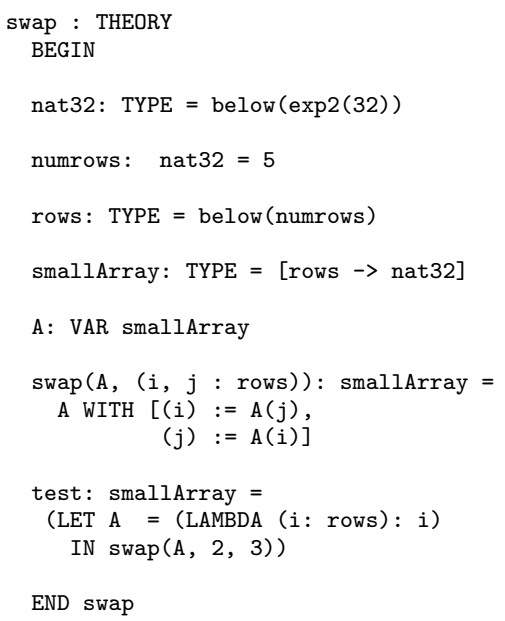

This generates the IR shown below. The two lookups of $A(j)$ and $A(i)$ are bound to the variables ivar_7 and ivar_10, respectively. The swap operation is made up of two updates. The variables are printed with their type information. Note that several of the variable occurrences are as arguments to the last operator. This operator marks the last occurrence of a variable in an evaluation path. It is used in the $\mathrm{C}$ translator for helping account for references. The whole definition is represented as a lambda expression where the body is given a return type following the arrow ' $\rightarrow$ '.

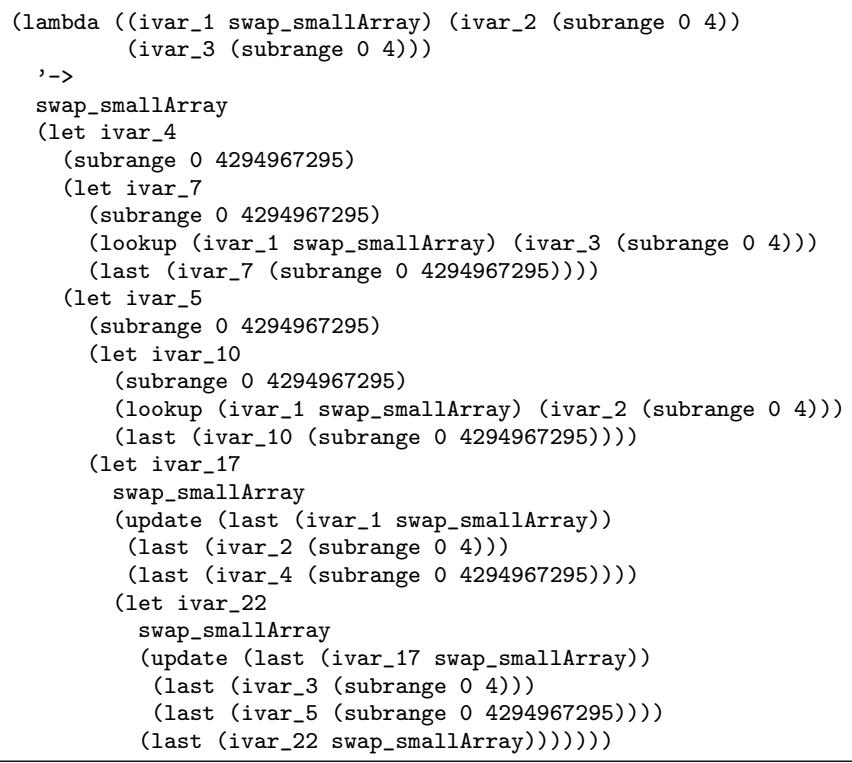

The operation ir2c generates the $\mathrm{C}$ counterpart of the IR translation. Two files: swap_c.h and swap_c.c are generated. The header file swap_c.h contains the following include declarations. 


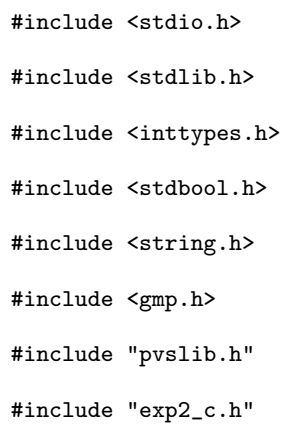

It also contains the type definition corresponding to the smallArray type. The array is defined by a struct that has a reference count field count, and the $\mathrm{C}$ array elems. We also define five operations for each such aggregate type: new, which constructs a fresh array; release, which decreases the reference count by one while freeing the struct if the reference count drops to zero; copy, which does a shallow copy; equal, which is a recursive equality test; and update, which performs an update.

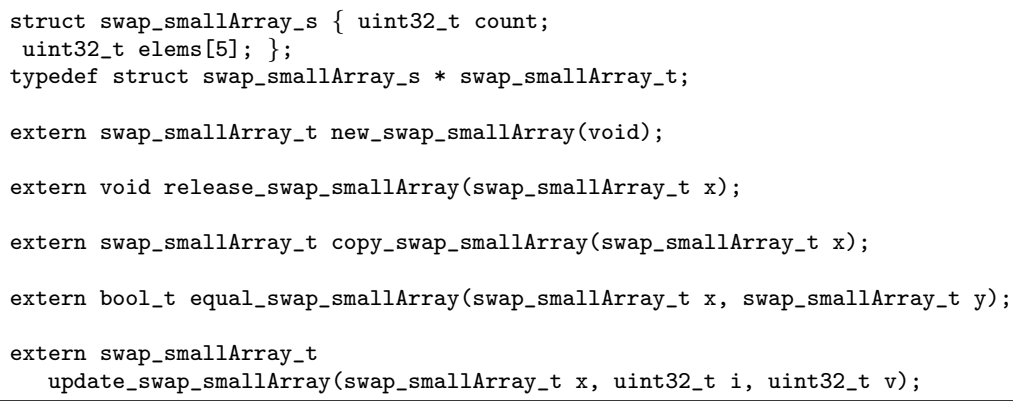

The file swap_c.c contains the definitions of the above operations, as well as the definition of swap. Each sub-expression in the IR definition of swap is translated with a return variable, where each LET-binding turns into an assignment with a possible casting. It might seem surprising that there is no explicit reference counting in the definition. The update operation manages the reference count for the array being updated. Since the two update operations are applied to variables marked as last, they will be executed in place if the reference count of the array passed into the operation as ivar_1 has a reference count of one.

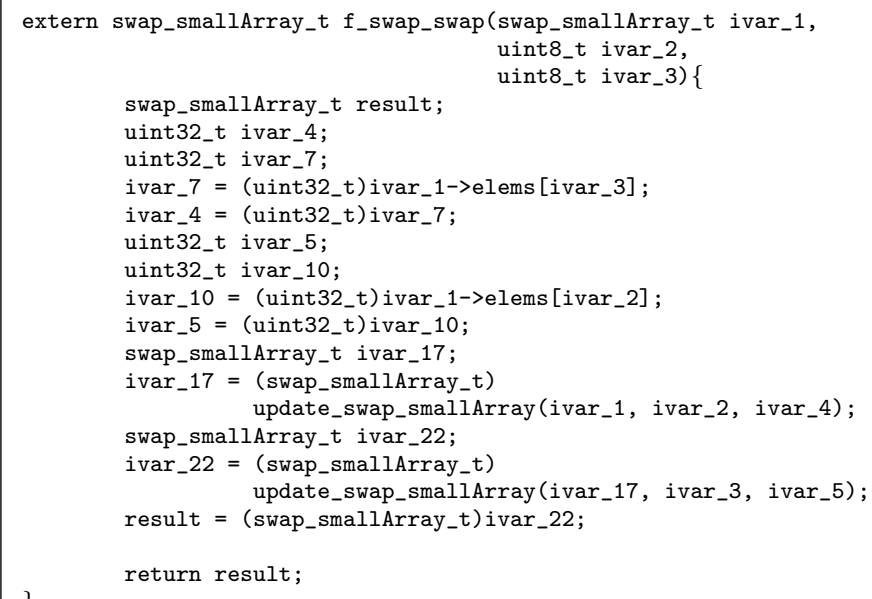


The generated programs can be tested by means of a hand-written main such as the one shown below.

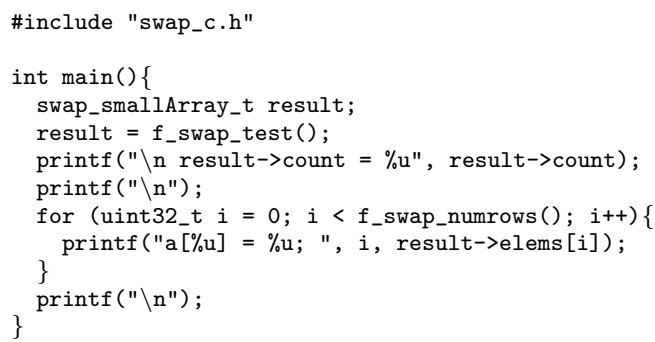

Compiling and executing this program generates.

result->count $=1$

$\mathrm{a}[0]=0 ; \mathrm{a}[1]=1 ; \mathrm{a}[2]=3 ; \mathrm{a}[3]=2 ; \mathrm{a}[4]=4 ;$

\section{Arithmetic Operations}

We next examine the translation of arithmetic operations. The theory arithops shows some of the types and a few variations on addition.

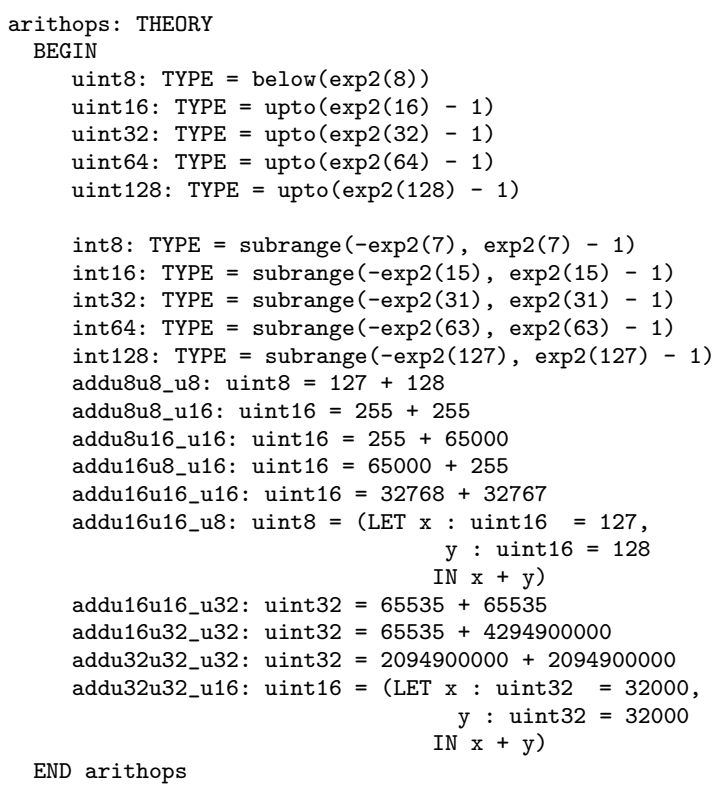

We show the generated code for the last operation addu32u32_u16. This code fragment illustrates the casting between the different numeric types. PVS2C handles casting between signed and unsigned 8-bit, 16-bit, 32-bit, 64-bit, and 128-bit numbers, as well as multi-precision representations. 


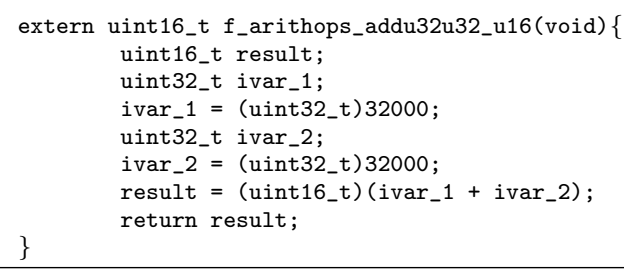

The multi-precision computations use the Gnu Multi-Precision (GMP) library. The operations employ a different calling convention where the first argument to the operation is the variable used for recording the result.

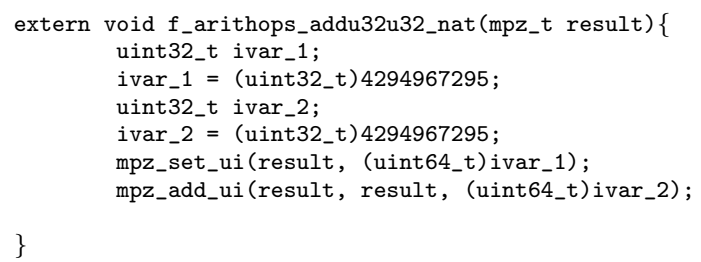

PVS record datatypes are represented as $\mathrm{C}$ structs with the corresponding fields. The PVS declaration for the record type smallPair is shown below as consisting of three fields: left, right, and mid.

smallPair: TYPE $=[\#$ left, right : smallArray, mid: nat32 \#]

The corresponding $\mathrm{C}$ type is shown below.

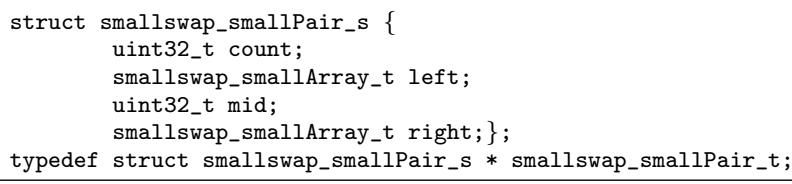

As with arrays, the record types also have five generated operations for creating a new object, copying an object, checking for equality, updating an object, and releasing the object.

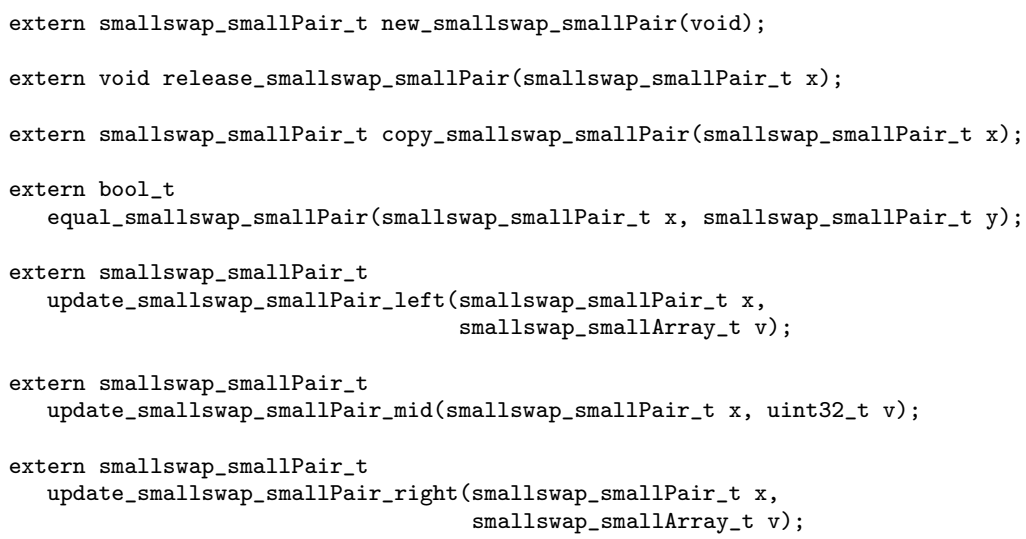

PVS $n$-tuples are treated as record types with fields project_1 to project_n.

Recursive datatypes in PVS are introduced with constructors along with their recognizers and accessors. The declaration for numlist introduces a datatype with two constructors: nnull 
and ncons, where nnull has no accessors and the recognizer nnull?, and ncons has two accessors: ncar and ncdr, and the recognizer ncons?.

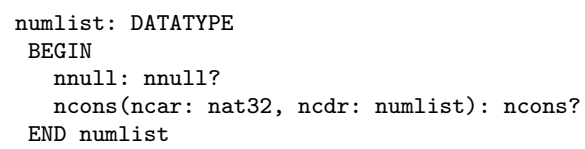

The translation to $\mathrm{C}$ first generates a parent datatype with just the count field for the reference count and an index field for marking the index of the constructor.

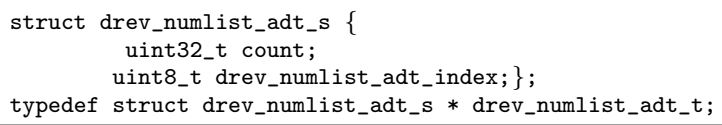

For each nontrivial constructor, there is a struct extending the parent struct with the relevant fields. For example, the constructor ncons yields the struct definition below extending the struct drev_numlist_adt_s.

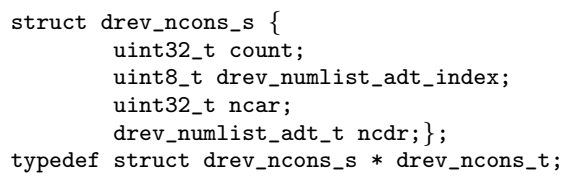

Datatype updates are handled in the same way as structs so that it is possible to define destructive counterparts for appending and reversing lists. PVS2C does not yet handle parametric datatypes nor parametric theories.

PVS2C handles closures by first generating a parent $\mathrm{C}$ struct for the function type with fields for

- The reference count

- The unary function pointer fptr

- The multiary function pointer mptr

- The release function pointer rptr

- The copy function pointer cptr, and

- A hashtable for storing function updates.

The corresponding $\mathrm{C}$ representation is defined below. The actual value representing a closure also contains a field for the bindings for the free variables.

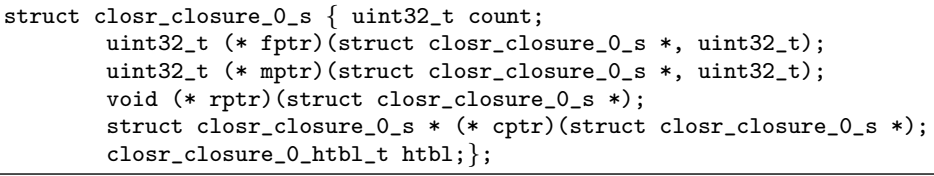




\section{Conclusions}

The PVS2C code generator translates an applicative fragment of PVS into C code. The generated $\mathrm{C}$ code is self-contained and does not rely on a run time. The generated code preserves the type safety of the typechecked PVS. It can only crash by exhausting resource bounds. The generated $\mathrm{C}$ code is comparable in efficiency to the corresponding hand-crafted $\mathrm{C}$, and a lot faster than the Common Lisp code generated from PVS.

We are working on extending the translation to cover parametric theories, dependently-sized arrays, and strings. We also plan to integrate it with the random testing capability in order to test the generated code on large sets of test vectors. The intermediate language is independently useful and we plan to support it with various forms of static analysis that can improve the quality of the generated code. Eventually, we would also like to handle specifications of concurrent systems so that we can generate monitors and entire systems starting from abstract specifications.

Acknowledgments. This material is based on work supported by NASA NRA NNA13AC55C, NSF Grant CNS-0917375, and by DARPA and the United States Air Force under agreement contract number FA8750-16-C-004315-C-0010. The views and conclusions contained herein are those of the authors and should not be interpreted as necessarily representing the official policies or endorsements, either expressed or implied, of NASA, NSF, USAF, DARPA, or the U.S. Government. We thank the anonymous referees for their constructive feedback.

\section{References}

[1] Gaspard Férey and Natarajan Shankar. Code generation using a formal model of reference counting. In Sanjai Rayadurgam and Oksana Tkachuk, editors, NASA Formal Methods: 8th International Symposium, NFM 2016, Minneapolis, MN, USA, June 7-9, 2016, Proceedings, pages 150-165, Cham, 2016. Springer International Publishing.

[2] Cormac Flanagan, Amr Sabry, Bruce F. Duba, and Matthias Felleisen. The essence of compiling with continuations (with retrospective). In Kathryn S. McKinley, editor, Best of PLDI, pages 502-514. ACM, 1993.

[3] Natarajan Shankar. Static analysis for safe destructive updates in a functional language. In A. Pettorossi, editor, 11th International Workshop on Logic-based Program Synthesis and Transformation (LOPSTR 01), Lecture Notes in Computer Science, pages 1-24. Springer-Verlag, 2002. Available at ftp://ftp.csl.sri.com/pub/users/shankar/lopstr01.pdf. 